\title{
REPENSANDO LAS MATERNIDADES: EL CASO DE PAREJAS LESBIANAS USUARIAS DE NUEVAS TECNOLOGÍAS REPRODUCTIVAS
}

\section{Rethinking maternities: The case of lesbian couples users of new reproductive technologies}

\author{
Noelia Soledad Trupa \\ noeliatrupa@hotmail.com \\ Universidad de Buenos Aires - Argentina
}

Recibido: $03-02-2017$

Aceptado: 06-06-2017

\section{Resumen}

En el presente artículo nos proponemos analizar desde una perspectiva de género la relación entre las maternidades en parejas lesbianas y las nuevas tecnologías reproductivas (NTR); y explorar el vínculo entre ambas en relación a la dicotomía naturaleza-cultura y sexo-género. A partir de estos dos ejes de análisis reflexionamos sobre las posibles situaciones y dilemas de parejas lesbianas que deciden ser madres y llevar a cabo su proyecto parental, a través de un tratamiento con NTR. Se trata de un análisis teórico-político en el cual partimos de definir y problematizar la categoría de género como principal instancia de estudio, para luego desarrollar los ejes propuestos en torno a la problemática planteada.

Palabras claves: Género, maternidades, lesbianas, nuevas tecnologías reproductivas.

\begin{abstract}
In the present article, we analyze, from a gender perspective, the relationship between maternity in lesbian couples and the new reproductive technologies (NRT); and explore the link between both in relation to the nature-culture and sex-gender dichotomy. From these two axes of analysis, we reflect on the possible situations and dilemmas of lesbian couples who decide to be mothers and carry out their parental project, through a treatment with NTR. This is a theoretical-political analysis in which we start to define and problematize the category of gender as the main study, to then develop the proposed axes around the problem raised.
\end{abstract}

Keywords: Gender, maternities, lesbians, new reproductive technologies. 


\section{Introducción}

En el presente artículo nos proponemos analizar desde una perspectiva de género la relación entre las maternidades en parejas lesbianas y las nuevas tecnologías reproductivas (NTR), y explorar el vínculo entre ambas en relación a la dicotomía naturaleza-cultura y sexo-género.

A partir de estos dos ejes reflexionamos sobre la situación de parejas lesbianas que deciden ser madres y llevar a cabo su proyecto parental, a través de un tratamiento con NTR. Buscamos desnaturalizar los significados asociados comúnmente a conceptos como naturaleza y cultura, sexo y género e indagar de qué manera tanto la interpretación social de los mismos como su uso se deriva de relaciones de poder que moldean dichos sentidos, legitimando ciertas prácticas y discursos y excluyendo otros.

\section{Discutiendo el concepto de Género}

Antes de comenzar el análisis, consideramos necesario definir qué entendemos por "género". Es una categoría de análisis transdisciplinaria que remite a rasgos psicológicos y socioculturales que se atribuyen a cada uno de los sexos en cada momento histórico y social. Las construcciones históricas de los géneros constituyen sistemas de poder que impregnan la vida social (De Lauretis, 1996; Scott, 1996; Butler, 2001). Adoptar una perspectiva de género, entonces, implica analizar las relaciones de poder entre los géneros, las cuales atraviesan todo el entramado social y se articulan con otras relaciones sociales, como las del sector socioeconómico, etnia, edad, orientación sexual y religión, entre otras.

Siguiendo el estudio de Joan Scott (1996), podemos decir que cuando hablamos de género nos referimos a esos comportamientos socialmente instituidos, aprendidos en función de nuestro sexo.

En "El tráfico de mujeres: notas sobre la "economía política” del sexo", Gayle Rubin (1986) define el concepto de sexo/género como "un conjunto de disposiciones por el que una sociedad transforma la sexualidad biológica en productos de la actividad humana" (1986: 30). Sin embargo, más adelante la autora reconoce que en dicho trabajo no distinguió el deseo sexual del género, tratando a ambos como modalidades del mismo proceso social subyacente (Rubin, 1989), lo que no es una formulación adecuada para el análisis de la sexualidad en las sociedades modernas occidentales; cuestión que retomaremos más adelante.

A su vez, desde una perspectiva antropológica, podemos afirmar que tanto el varón como la mujer son productos culturales, es decir, son construidos socialmente y definidos en torno a un “otro". Es así que la mujer se define por oposición al varón y viceversa, con lo cual las características y modos de actuar que se esperan de la mujer serán muy disímiles a los que se 
asocian a la "masculinidad" y se esperan de los varones, llevando a una naturalización de los roles y funciones en base al sexo. Sin embargo, cabe aclarar que es la mujer la que se define en función de un "otro", porque ella es el sujeto subordinado en las relaciones entre los géneros. Pero lo que sí ocurre con ambos es que se recortan en el juego de las múltiples diferencias que atraviesan los géneros, y el modo de procesar esas diferencias dependerá de las diversas culturas. A ello se refiere Sherry Ortner en su artículo “¿Es la mujer con respecto al hombre lo que la naturaleza con respecto a la cultura?" (1979), cuando sostiene que la subordinación de género no puede analizarse sólo a partir de un seguimiento de los roles y funciones desempeñadas por cada uno de los sexos, sino a través de toda una serie de significaciones, símbolos y sentidos asociados a los mismos. Es decir, que el problema central no son las tareas desarrolladas por cada uno de ellos, sino los significantes y valoraciones asociadas a las mismas. Ortner sostiene cómo todas las culturas tienen diversas valoraciones culturales y buscará demostrar cómo las culturas consideran inferiores a las mujeres a partir de tres tipos de datos:

"1-elementos de la ideología cultural y declaraciones de los informadores que explícitamente desvalorizan a las mujeres concediéndoles a ellas, a sus funciones, a sus tareas, a sus productos y a sus medios sociales, menos prestigio que el concedido a los hombres y a sus correlatos masculinos; 2artificios simbólicos, como el atribuirles una cualidad contaminante, que debe interpretarse con el contenido implícito de una afirmación de inferioridad; y 3-los ordenamientos socioestructurales que excluyen a la mujer de participar o tener contacto con determinadas esferas donde se supone que residen los poderes sociales" (1979:4). Para la autora ello es prueba suficiente de la subordinación universal de las mujeres. Precisamente, su tesis es "que la mujer ha sido identificada con [...] algo que todas las culturas desvalorizan, algo que todas las culturas entienden que pertenece a un orden de existencia inferior a la suya", y además sostiene que, al parecer, "sólo hay una cosa que corresponda a esta descripción, y es la naturaleza en su sentido más general" (Ortner, 1979: 6).

En este sentido, nos dirá que la cultura estará asociada a la conciencia humana y a las producciones de la misma, mediante las cuales se controlará a la naturaleza. Si bien tanto la cultura como la naturaleza son categorías conceptuales, nos permiten pensar las relaciones entre hombres y mujeres en términos de inferioridad y superioridad.

Siguiendo a la autora, podemos decir que los hechos biológicos y "naturales" no es que "sean irrelevantes ni que hombres y mujeres no sean distintos, sino que estos hechos y diferencias sólo adoptan la significación de superior/inferior dentro del entramado culturalmente definido del sistema de valores" (Ortner, 1979: 5).

Michelle Rosaldo hace un análisis similar en "Mujer, cultura y sociedad: Una visión teórica" (1979), donde estudió el rol y status de las mujeres, y nos dice que en relación a los varones, ellas carecen de una autoridad universalmente reconocida. La autora retoma los trabajos de Margaret Mead para demostrar que las características asociadas a lo "femenino", como la pasividad, el conformismo, la debilidad y la complacencia en el cuidado de los niños, dependerá de las características de cada tribu en particular; con lo cual no existe eso que consideramos "femenino" 
como algo innato e inmutable en las mujeres, ya que estos pueden ser rasgos que caractericen también a los varones. Es decir, que cualquier comportamiento es variable y no puede ligarse exclusivamente al sexo. Sin embargo, nos dice la autora que "todas las sociedades conocidas reconocen y elaboran algunas diferencias entre los sexos" (Rosaldo, 1979:2), ya sea en el temperamento, en la personalidad y/o en la contextura física. Pero lo que es más llamativo para Rosaldo, al igual que para Ortner, no son las diferencias asociadas a los sexos, sino las valoraciones que conllevan cada uno de ellos. Por ejemplo, las actividades desarrolladas por varones son consideradas más importantes y prestigiosas, ante lo cual Rosaldo sostiene que "aparece como universal una asimetría en las estimaciones culturales de los hombres y de las mujeres" (1979: 3), ya sean de tipo económicas, sociales, culturales y/o políticas. La autora también sostiene "que en todas partes los hombres tienen una autoridad sobre las mujeres, que tienen un derecho -culturalmente legitimado- a la subordinación y sumisión de éstas" (Rosaldo, 1979: 5). No obstante, ello no implica negar la importancia de la mujer, pero Rosaldo sostiene "que la asimetría característica de la experiencia de los hombres y de las mujeres [...] puede entenderse en términos no directamente biológicos, sino de un hecho casi universal en la experiencia humana" (1979: 6), enunciado al cual también adhiere Ortner. El hecho de que, "en la mayoría de las sociedades tradicionales, las mujeres pasan una buena parte de su vida de adultas pariendo y cuidando a sus hijos, lleva a una diferenciación de los terrenos de la actividad que se concreta en doméstica y pública; puede tenerse en cuenta [...] para aclarar una serie de aspectos importantes de la estructura social y psicológica humanas" (Rosaldo, 1979: 7). Esa oposición entre público y doméstico (o privado) nos ayudará a comprender el porqué de los roles y situaciones determinadas en que se encuentran cada uno de los sexos, ya sea en sus aspectos económicos, psicológicos, culturales y sociales.

\section{La(s) mujer(es) y la(s) maternidad(es)}

En el caso de las mujeres, su rol reconocido como el más importante socialmente es el de ser madres, con lo cual el resto de sus actividades se hallan condicionadas por la tarea del cuidado de los niños y el pasar la mayor parte del tiempo en el ámbito doméstico (Rosaldo, 1979). Desde este punto de vista, la asociación de la mujer con la naturaleza, empieza con su cuerpo y su función procreadora. Ortner señala, retomando el análisis de Simone de Beauvoir en El Segundo Sexo (1953), que "una mayor parte del cuerpo femenino, durante un mayor período de su vida, y con un cierto- a veces gran- costo de su salud personal, fuerzas y estabilidad general, se ocupa de los procesos naturales relativos a la reproducción de la especie” (1979: 10).

Además, nos interesa rescatar esta idea aceptada socialmente de que "una mujer se convierte en una mujer si sigue los pasos de su madre" y que "a las mujeres se las concibe casi exclusivamente como hermanas, esposas y madres" (Rosaldo, 1979: 11). 
En relación con ello, nos preguntamos qué ocurre en el caso de las parejas lesbianas que desean y llevan a cabo su proyecto de ser madres: ¿no ponen en cuestionamiento estos valores ideales asociados a la maternidad en el seno de una pareja heterosexual?, ¿no rompen con ciertos estereotipos culturales asociados a la ética maternal?, ¿ello no es profundizado por el tratamiento a partir de las NTR? Estos son algunos de los interrogantes acerca de los cuales nos proponemos pensar la relación entre la maternidad lésbica y las NTR, porque además pone en cuestión esa división tan tajante entre naturaleza y cultura, para pensar las relaciones de género. Porque, si como dijimos antes, el status de las mujeres "deriva del estadio del ciclo de la vida en que se encuentren, de sus funciones biológicas, $\mathrm{y}$, en particular, de sus lazos sexuales y biológicos con hombres en concreto" (Rosaldo, 1979: 14), ello también ¿no puede ser problematizado en el caso de las lesbianas?

Es decir, que las "nociones culturales sobre la mujer gravitan a menudo en torno a sus características naturales o biológicas: fertilidad, maternidad, sexo y flujo menstrual" y "la pureza y corrupción son ideas que se relacionan sobre todo con las mujeres que deben negar su cuerpo o limitar su peligrosidad sexual" (Rosaldo, 1979: 14). Siguiendo este análisis de Rosaldo, podemos decir que a las lesbianas se las considera socialmente como "anómalas", ya que no se ajustan a las características definidas como propias de la "feminidad" y a los roles que se esperan de ellas en función de su sexo, es decir, en su condición de "mujeres".

Entonces, desde la perspectiva de ambas autoras podemos sostener que se equipara a la mujer (y su cuerpo) con la naturaleza, en oposición a los hombres asociados al mundo de la cultura. La mujer es pensada como subordinada y relegada al espacio doméstico como ámbito no perteneciente a la cultura, y cuya función biológica (natural) más valorada es el ejercicio de la maternidad. No obstante, entendemos esta última como una experiencia subjetiva y una práctica social, es decir, es una construcción cultural, histórica y política (Tubert, 1991, 1996; Schwarz, 2007, 2010). Concebirla sólo como un hecho biológico implica adherir a una imagen totalizadora y unificada de la mujer = madre (Tubert, 1996; Fernández, 1993; Schwarz, 2007, 2010). Esta concepción de la maternidad como esencia de la mujer y su consecuente creencia en la existencia del instinto materno, son funcionales a la división sexual del trabajo que históricamente ha formado parte de la normativa de género, que legitima y naturaliza la subordinación de la mujer en relaciones asimétricas de poder, como vimos anteriormente.

Es decir que, en general, el ideal maternal (mujer = madre) es entendido, desde diversos estudios de género, como una forma de subordinación de las mujeres. Al asociarse la mujer con la naturaleza, se asocia ese ideal maternal al destino biológico de las mujeres, relegándolas al ámbito doméstico y privado, en contraposición al espacio público propio de los hombres. Si bien esta división sexual del trabajo ha entrado en crisis, ya que ambos espacios no pueden pensarse como totalmente distintos y separados; todavía sobreviven estudios (como el de Rosaldo) que remarcan esa división como central en la normativa de género. 
Pero proponemos otra lectura de estos procesos: ¿por qué no pensar el ejercicio de la maternidad también como un espacio de resistencia a la normativa de género? En este sentido, si como sostienen Ortner y Rosaldo, podemos pensar a la madre como el agente de la primera socialización de los niños/as, y así pensar estas funciones de cuidado y transmisión de saberes en conexión con la cultura, más allá de que se produzcan en el espacio doméstico, ¿no podemos pensar también este último como un espacio de resistencia?, donde se produzcan la transmisión de otros saberes por parte de estas madres lesbianas, que logren ir desarmando los estereotipos de género legitimados socialmente. No necesariamente ello tiene que ocurrir, pero es una posibilidad.

\section{Sexualidad y Reproducción: Madres lesbianas}

El objetivo de este trabajo es entonces problematizar la subordinación de las mujeres a partir del ideal maternal y pensar la maternidad como un espacio de relaciones de poder, de disputas, donde no hay sólo subordinación sino también resistencias a partir del ejercicio de la maternidad, sobre todo en parejas lesbianas donde ser madres puede ser visto socialmente como una transgresión. Autoras como Gabriela Malaguera González (2008) han hecho esta lectura, y sostienen que para el pensamiento heterosexual hegemónico reproducir la especie es reproducir la heterosexualidad, con lo cual mientras que ser madre desde la heterosexualidad puede ser una de las maneras de asimilarse a la cultura dominante, el deseo de un hijo/a en las parejas lesbianas puede ser un deseo subversivo.

Para seguir reflexionando acerca de estas cuestiones, y sin reificar el lesbianismo, nos resulta interesante explorar en la dimensión de sexualidad para profundizar el análisis.

Retomando el análisis de Michel Foucault en Historia de la Sexualidad (2009), podemos decir que la sexualidad no se refiere propiamente a algún atributo de los cuerpos; es una producción cultural: representa la apropiación del cuerpo humano y de sus capacidades fisiológicas por un discurso ideológico. La sexualidad no es una cosa, un hecho natural e inmóvil en la subjetividad humana, sino el juego de efectos producidos en los cuerpos, conductas y relaciones sociales por un despliegue de una tecnología política compleja (Foucault, 2009). Consideramos a la sexualidad en sentido amplio, no meramente restringida a la genitalidad, sino referida también a la emocionalidad, la afectividad y la subjetividad, entre otros aspectos. Ello es lo que también afirma Gayle Rubin (1989) cuando dice que no es que "las capacidades biológicas no sean prerrequisitos de la sexualidad humana" [...] sino que esta última "no puede comprenderse en términos puramente biológicos" (1989: 15). Esta autora señala además del "esencialismo sexual", otros axiomas problemáticos en torno a la sexualidad como son: "la negatividad sexual, la falacia de la escala extraviada, la valoración jerárquica de los actos sexuales, la teoría del 
dominó del peligro sexual y la ausencia de un concepto de variedad sexual benigna" (1989: 16). Si bien no entraremos en detalles acerca de qué trata cada uno de ellos, creemos fundamental para comprender más en profundidad el tema de este trabajo, retomar el axioma de las jerarquías sexuales, es decir, el sistema de valores sexuales que permea las relaciones sociales; según el cual, "la sexualidad buena, normal y natural sería idealmente heterosexual, marital, monógama, reproductiva y no comercial" (Rubin, 1989: 21). Es decir, que "cualquier sexo que viole estas reglas es malo, anormal o antinatural. El sexo malo es el homosexual, promiscuo, no procreador, comercial o el situado fuera del matrimonio" (Rubin, 1989: 21). Es interesante pensar la situación de las parejas lesbianas que recurren a las NTR para ser madres, dentro de este esquema, ya que estarían desafiando el ideal de sexualidad presente en la sociedad.

Mientras para el pensamiento religioso el ideal es la pareja heterosexual casada que puede procrear, para la psicología es la heterosexualidad madura. Estos constituyen algunos de los saberes legítimos en nuestra sociedad, que en términos de Foucault, producen relaciones de poder, frente a las cuales las parejas homosexuales ${ }^{1}$ y sus proyectos parentales quedarían excluidos, ya que se alejan del ideal hegemónico.

En este sentido, Rubin concluye, como remarcamos al comienzo de este trabajo, que "aunque el sexo y el género están relacionados, no son la misma cosa, y constituyen la base de dos áreas distintas de la práctica social" y "es esencial analizar separadamente género y sexualidad si se desean reflejar con mayor fidelidad sus existencias sociales distintas" (1989: 54). Es decir, que a diferencia de lo que piensan algunas feministas, la sexualidad no es una simple derivación del género.

De este modo, podemos pensar que, en el caso de las lesbianas, no sólo pueden considerarse como oprimidas por su condición de mujeres (género), sino también por su orientación sexual (sexualidad), debido entre otras cosas, a esta idea de "estratificación sexual" analizada anteriormente. Sin embargo, cuestionamos la idea de una opresión total, ya que por ejemplo, a partir de su elección de ser madres a través de las NTR, esta también puede ser leída como una doble transgresión: tanto por ser madres lesbianas como por hacerlo a través de las nuevas tecnologías; resultado del efecto paradójico que conlleva el uso de las mismas, "que al mismo tiempo que refuerzan el ideal maternal tradicional, socavan las bases de la familia tradicional, al fragmentar la maternidad en múltiples componentes y hacer posibles modelos familiares nuevos" (Tubert, 1996: 36).

Esta lectura nos lleva a problematizar el axioma de la universalidad de la subordinación de las mujeres, además de realizar una crítica a pensar las categorías en términos exclusivamente

\footnotetext{
${ }^{1}$ Nos referiremos a las personas homosexuales como aquellas "que sienten deseo hacia las de su mismo sexo, sin intentar adoptar la apariencia física del sexo opuesto. El sustantivo y adjetivo 'homosexual' se utilizan de modo genérico, en tanto que el sustantivo y adjetivo 'gay' o 'lesbiana' se utilizan más precisamente para referirse a los individuos homosexuales que asumen con cierto grado de publicidad su orientación sexual" (Pecheny, 2002: 127).
} 
binarios (naturaleza-cultura; sexo-género), ya que los mismos terminarían reificando no sólo dichas categorías y el análisis de los procesos que suelen ser contradictorios y complejos, sino también reificando las identidades, ya sean culturales, políticas y/o sexuales. De ello también habla Verena Stolcke (1999) y será retomado cuando pensemos más cabalmente acerca de las posibilidades para ser madres que ofrecen las NTR.

Incluso la misma Sherry Ortner (2006), casi tres décadas después de publicado su artículo “¿Es la mujer con respecto al hombre lo que la naturaleza con respecto a la cultura?”, revisa algunos de sus postulados sobre la universalidad del patriarcado y de la oposición naturaleza/cultura. Ella afirma que su error más importante fue sostener que la vinculación entre mujer y naturaleza, hombre y cultura, "explica” la dominación masculina, sea esta universal o no. Ahora bien, la autora afirma que la explicación de esta última le parece más adecuada "como el resultado de una compleja interacción de disposiciones funcionales, dinámicas de poder, y factores corporales" (Ortner, 2006: 17).

Otro problema, como dijimos anteriormente, es la supuesta universalidad e inmutabilidad en los significados y sentidos de categorías de análisis como las de naturaleza y cultura. Es decir, que la concepción de cada uno de estos términos y la valoración asociada a los mismos diferirá de acuerdo al momento histórico, los sujetos que se estén analizando y desde qué perspectiva se efectúe dicho análisis. Y, como afirma Ortner, "esta variación en el nivel de los significados explícitos culturales [...] es de hecho fundamental en la construcción del género y la sexualidad desde una perspectiva transcultural" (2006: 17). De allí, la importancia de discutir estos dilemas conceptuales, teóricos y políticos. En este sentido, Ortner señala cómo el género es una de las herramientas más poderosas para analizar categorías como cultura y/o naturaleza; y a la inversa, el lenguaje de estas últimas nos permite examinar tanto la sexualidad, la reproducción, como las relaciones de poder presentes en cada cultura.

Retomando el análisis, y como decíamos previamente, si bien las lesbianas en su condición de "mujeres" estarían capacitadas socialmente para ejercer la maternidad, su orientación sexual las excluiría de la misma. Como sostiene Schwarz, "esta concepción está marcada por los estereotipos sociales sobre la homosexualidad que suponen que la orientación sexual de las madres influirá en las elecciones sexuales del niño, que éste tendrá una identidad sexual poco clara o impropia o que estigmatizarán al niño en la escuela o en sus relaciones” (2008: 1). Si bien podemos pensar que en el reciente marco de sanción de la Ley de Matrimonio Igualitario y actual reforma del código civil en Argentina, se estaría avanzando en la aceptación y el respeto a las parejas homosexuales y sus proyectos parentales, contribuyendo de a poco a una ampliación de sus derechos y un mayor reconocimiento y protección a la diversidad familiar; no podemos dejar de negar el enorme peso y control que ejerce todavía la heterosexualidad obligatoria y la normativa asociada a la ética maternal en nuestras prácticas y representaciones sociales. 


\section{El acceso a las Nuevas Tecnologías Reproductivas de parejas lesbianas}

Por otra parte, podemos afirmar como "la aceptación de la homosexualidad (como la de la anticoncepción y la del aborto), supone la legitimidad de la disociación entre sexo y procreación" (Pecheny, 2002 en Schwarz, 2008). Si bien esta separación es un proceso que comenzó hace un tiempo, admite una serie de transformaciones; algunas de las cuales se profundizan a partir de la existencia de nuevas tecnologías en el campo de la medicina reproductiva. Entendemos las NTR como el "conjunto de técnicas que desde el campo interdisciplinario de la medicina terapéutica o de intervención y la medicina experimental, se proponen como una respuesta, más o menos efectiva en términos de sus resultados, a la ausencia de hijos no voluntaria de individuos o parejas" (Ariza, 2007: 257). Nos resulta interesante analizar cómo las NTR forman parte de la lógica biomédica de intervención, modificación y perfeccionamiento de los cuerpos, en torno a la cual se produce un complejo debate ético - ideológico; no sólo en relación con las consecuencias sociales de la aplicación de estas técnicas médicas, sino también de sus implícitos valores eugenésicos. Entendemos por eugenesia "la ciencia y el arte que tratan de mejorar la constitución genética de los seres humanos" (Taboada, 1986: 9).

Si bien, estas nuevas tecnologías rompen, por un lado, con la asociación entre sexualidad y reproducción; por otro, desafían esa supuesta separación entre naturaleza y cultura, constituyendo a los cuerpos como una especie de híbridos, en palabras de Donna Haraway (1995), de "cyborgs". La autora propone este concepto como un "recurso imaginativo" frente a un pensamiento y un mundo dicotomizado que excluye y uniformiza a las mujeres. El "cyborg" es una ficción que pretende abarcar la realidad social y corporal de las mujeres, como una forma de abordar la ruptura de los dualismos, que en la cultura científica actual se concreta en la discusión de las fronteras entre lo humano y lo animal, lo humano y la máquina, lo físico y lo no físico.

Esta relación entre lo humano y la máquina nos resulta especialmente interesante en el caso de las parejas lesbianas que recurren a dichos tratamientos (como la inseminación artificial o la recepción de óvulos de su pareja), ya que la experiencia de la maternidad para estas parejas usuarias de las NTR implica profundas transformaciones en los valores, creencias y representaciones tradicionales y comúnmente aceptadas acerca del ideal maternal. Una de ellas, tal vez la más importante, es esta separación entre sexualidad y reproducción, la disociación entre la concepción y la filiación, la filiación biológica y los vínculos afectivos, poniendo en cuestión significados habitualmente otorgados a conceptos como familia, pareja, maternidad, paternidad, hijo/a, entre otros (Tubert, 1991).

En este sentido, Stolcke también realiza una crítica del dualismo moderno entre naturaleza y sociedad, por ejemplo, retomando los estudios en el campo de la biotecnología y la biogenética como desafiantes de la disociación entre ambas. 
Stolcke critica el sentido común occidental que considera la naturaleza como separada de la cultura, como dos aspectos distintos de la experiencia humana. En este sentido, problematiza la noción biologista, naturalista de la función de las mujeres en la cultura occidental, y analiza qué tiene que ver la clase y la raza con ello. Si bien estas últimas no serán analizadas en este trabajo, me resulta clave resaltar que tanto la etnia como la clase social son dimensiones de análisis fundamentales para comprender las relaciones de género, ya que no es lo mismo ser una mujer heterosexual, de clase media y blanca, que ser una mujer lesbiana, de clase baja, negra u oriental. Como bien sostiene la autora, "las diferencias de sexo no menos que diferencias de raza son construidas ideológicamente como "hechos" biológicos significativos en la sociedad de clases, naturalizando y reproduciendo así las desigualdades de clase. Es decir, se construyen y legitiman las desigualdades sociales y de género atribuyéndolas a los supuestos "hechos biológicos" de las diferencias de raza y sexo" (Stolcke, 1999: 42).

En especial, del estudio de Stolcke nos interesan particularmente algunas cuestiones como, por ejemplo, esta idea de cómo "las doctrinas biologistas de la desigualdad han contribuido también a consolidar la noción genética de la familia como célula biológica natural y por lo tanto universal básica de la sociedad. Estas han fomentado una idea individualizada y biológica de la maternidad y de la paternidad, es decir, de los vínculos entre padres e hijos como "lazos de sangre" [...] Un componente de este sistema de valores atravesado por criterios biológicos son las ansias de inmortalidad, en particular por parte de hombres, que se plasman en el fuerte deseo de reproducir sus genes a través de las generaciones y la imagen, complementaria, de las mujeres como "destinadas" por su biología a la maternidad y a la domesticidad al servicio de éstos" (1999: 49). Sin embargo, la tradicional familia nuclear monogámica se está descomponiendo, dando cuenta de ello la cada vez mayor diversidad familiar.

Además, y en relación con estos cambios, nos preguntamos si existe una vinculación entre la importancia otorgada a las NTR en la sociedad actual y la consolidación del ideal maternal (mujer $=$ madre $)$ como realización plena de las mujeres; y en ese caso, nos interrogamos acerca de la situación de las mujeres lesbianas que acceden a dichos tratamientos, donde la maternidad puede ser buscada como una reivindicación de su "condición de mujer" y su derecho a ser madre, como una transgresión al ideal de madre heterosexual o como deseo/realización personal. En este sentido, las NTR ponen en cuestión la idea de "la biología es destino", ya que, en el caso de las parejas lesbianas, más allá de la imposibilidad reproductiva de su práctica sexual, pueden llevar adelante su proyecto parental, a partir, por ejemplo, de la donación de óvulos por parte de una de las mujeres de la pareja, transformándose una de ellas en la madre biológica y la otra en la madre genética. Esto produce cambios, rupturas y algunas continuidades en las relaciones de parentesco y en la manera de experimentar los cuerpos, la maternidad y los nuevos modelos de familias. Podemos hablar entonces de homoparentalidad, monoparentalidad y parentalidad adoptiva, entre otras posibilidades. 


\section{A modo de cierre(s) y de apertura(s)}

Para finalizar, queremos resaltar que, en el presente análisis, no intentamos más que abrir y continuar algunas líneas de pensamiento en relación a la maternidad, el parentesco y las nuevas formas de llevarlos a cabo; haciendo especial hincapié en la importancia de lograr una visión más holista y dinámica de la naturaleza en relación a la cultura, no pensarlas como dos dimensiones separadas, sino en permanente cruce y tensión. En esta misma línea, siguiendo a Teresa de Lauretis (1996), podemos afirmar el fin del sujeto unitario, ya que este último tiene posiciones múltiples y está atravesado por discursos y prácticas que hasta incluso pueden ser contradictorios. Dentro de este marco, proponemos pensar el lugar de la mujer y del varón como posiciones intermedias entre la naturaleza y la cultura.

Podemos concluir que unas concepciones y significados culturales distintos sólo podrán surgir de una realidad social diferente, y viceversa. No sólo debemos transformar las instituciones sino también el lenguaje y los imaginarios sociales en pos de un incremento en la valorización de las mujeres en nuestras sociedades; y no terminar infiriendo a partir de nuestros análisis siempre una subordinación femenina en términos dicotómicos y binarios. Este es uno de nuestros mayores desafíos.

\section{BIBLIOGRAFÍA}

- Ariza, Lucía (2007): “Tecnologías reproductivas en la Argentina contemporánea: la experiencia de la infertilidad en mujeres usuarias". En: López, E. y Pantelides, E. (comp.) Aportes a la investigación social en salud sexual y reproductiva. Buenos Aires: CENEP, CEDES, AEPA, UNFPA, pp. 257-283.

- Butler, Judith (2001): El género en disputa. El feminismo y la subversión de la identidad. Buenos Aires: Paidós.

- De Beauvoir, Simone (1953): Le Deuxième Sexe. Francia: Gallimard.

- De Lauretis, Teresa (1996): La tecnología del género. En: Revista Mora, vol. 12, Buenos Aires, pp. 6-34.

- Fernández, Ana María (1993): La mujer de la ilusión. Pactos y contratos entre hombres y mujeres. Buenos Aires: Paidós.

- Foucault, Michel (2009): Historia de la Sexualidad 1: la voluntad de saber. Buenos Aires: Siglo Veintiuno Editores.

- Haraway, Donna J. (1995): Ciencia, cyborgs y mujeres. La reinvención de la naturaleza. Madrid: Ediciones Cátedra. 
- Malaguera González, Gabriela (2008): "Maternidad lesbiana: ¿posibilidad para la transgresión?”. En: Seminario Contranatural. La perspectiva teórico política de la diversidad sexual de la Maestría de Estudios de la Mujer de FACES/UCV, pp.32-55.

- Ortner, Sherry (1979): “¿Es la mujer con respecto al hombre lo que la naturaleza con respecto a la cultura?". En: Olivia Harris y Kate Young (comp.): Antropología y feminismo. Barcelona: Editorial Anagrama, pp. 109-131.

. (2006): "Entonces, ¿Es la mujer al hombre lo que la naturaleza a la cultura?”. En:

Antropólogos Iberoamericanos en Red, vol. 1, $\mathrm{n}^{\circ}$. 1, enero-febrero, pp. 12-21.

- Pecheny, Mario (2002): "Identidades discretas". En: Arfuch, Leonor (comp.): Identidades, sujetos y subjetividades. Buenos Aires: Prometeo Libros, pp.125-148.

- Rosaldo, Michelle (1979): "Mujer, Cultura y Sociedad: Una visión teórica”. En: Olivia Harris y Kate Young (comp.): Antropología y feminismo. Barcelona: Editorial Anagrama, pp. 1-54.

- Rubin, Gayle (1986): "El tráfico de mujeres. Notas sobre la "Economía Política" del sexo". En: Revista Nueva Antropología, vol. VIII, n. 30, Universidad Nacional Autónoma de México, pp.95-145.

(1989): "Reflexionando sobre el sexo: notas para una teoría radical de la sexualidad". En: Carole Vance (comp.): Placer y peligro. Explorando la sexualidad femenina. Madrid: Ed. Revolución, pp. 269-319.

- Schwarz, Patricia (2007): "Prácticas, estrategias y percepciones de la maternidad en mujeres jóvenes de clase media urbana”. En: Ana Lía Kornblit (coord.): Juventud y vida cotidiana. Buenos Aires: Ed. Biblos, pp. 52-65.

(2008): "Las lesbianas frente al dilema de la maternidad". En: Mario Pecheny, Carolos y Daniel Jones (comp.): Todo sexo es político. Estudios sobre sexualidades en Argentina. Buenos Aires: Ed. El Zorzal, pp. 193-214.

(2010): Maternidades e identidades de género. Prácticas y percepciones de mujeres de sectores socioeconómicos medios de la Ciudad Autónoma de Buenos Aires. Tesis doctoral en Ciencias Sociales (UBA). Mimeo. Buenos Aires.

- Scott, Joan (1996): “El género: una categoría útil para el análisis histórico”. En: Marta Lamas (comp.): El género: la construcción cultural de la diferencia sexual. México: PUEG, pp. 37-75.

- Stolcke, Verena (1999): “¿Es el sexo para el género como la raza para la etnicidad?”. En: Cuadernos para el Debate, $\mathrm{n}^{\circ}$. 6, IDES, pp. 26-60.

- Taboada, Leonor (1986): La maternidad tecnológica. De la inseminación artificial a la fertilización in Vitro. Barcelona: Icaria Editorial.

- Tubert, Silvia (1991): Mujeres sin sombra. Maternidad y tecnología. Madrid: Siglo Veintiuno. (1996): Figuras de la madre. Madrid: Ediciones Cátedra. 